\title{
Deploying FTTH with Distributed Control and Bus Topology
}

\author{
Rong Nan Chiou \\ *First-Third Department, First-Third University, Address Including Country Name \\ Department of Electrical Engineering, Koa Yuan University, Kaohsing City, Taiwan \\ rnchu@ms63.hinet.net
}

\begin{abstract}
In order to enhance the bandwidth utility and lower constructing and maintaining costs of public access networks, this paper introduces bus FTTH networks in order to deploy FTTH network with distributed control and bus topology.

After the unfair-access problem on optical-bus distributedcontrol TDMA networks had been completely resolved, a bus FTTH network consisting of two optical-bus distributed-control TDMA networks was presented. This paper compares bus FTTH networks with FTTH EPONs. Bus FTTH networks applied distributed-control mechanisms to control access in order that the merit of FTTH is evidently revealed. FTTH EPONs adopts centralized-control mechanisms which consume and waste bandwidth so that the merit of FTTH networks is debased. Otherwise, the constructing and maintaining costs of bus FTTH networks are much lower than FTTH EPONs. Therefore, bus FTTH networks are appropriate to be adopted in an all-fibre optical access environment in which every home is attached to a unit of optical fibres regardless of the size of the required bandwidth of homes.
\end{abstract}

Keywords - Distributed control, fibre to the home, optical-fibre communication, time-division multiple access, medium access control protocols.

\section{INTRODUCTION}

According to the strong demand on the quality of service from customers in recent years, the required bandwidth on access networks is exponentially increasing. Because the provided bandwidth, transmissible distance and network performance on traditional access networks are subject to twisted pairs, network managers have being enforced to develop the fibre-to-the-home (FTTH) network [1-2]. The deployment of FTTH networks is taking off in several regions [3-5], but the considerable concern about the high constructing and maintaining costs is the bottleneck of rapid and world-wide FTTH establishment [6-9]. Therefore, it is an important topic to explore how to suppress the constructing and maintaining costs for deploying FTTH networks.

Due to commercial behaviour, customers pay for the required quality of service. The more the payment, the higher the quality of service. So, public networks must provide a constant quality for service regardless of network load [10]. Otherwise, in order to keep the constant quality from varying with nodal positions, a public network must be inherent in the ideal fair behaviour of networks [11]. The ideal fair behaviour represents that a node within networks must experience the same performance when its nodal position is moved but its load is not changed [11]. FTTH networks are public access networks. Therefore, how to control an optical network to give rise to the ideal fair behaviour is another important topic for deploying FTTH networks.

Medium-sharing networks naturally have high bandwidth utility. Their topologies are so simple that low constructing cost will be taken easily. Many medium access control (MAC) protocol, such as carrier sense multiple access with collision detection (CSMA/CD), token ring, token bus, time-division multiple access (TDMA), and so on, is exploited by mediumsharing networks to control access among nodes. The TDMA protocol is appropriate for supporting optical medium-sharing networks. On TDMA networks, packet collisions can disappear. Therefore, the bandwidth utility of TDMA networks can approximate to its medium capacity. However, the optical TDMA network has an unfair-access problem due to its bus topology [12]. On the optical-bus TDMA networks, every node directly connecting with the bus controls access by itself. In a word, an optical-bus distributed-control TDMA network has the unfair-access problem. The unfair problem indicates that the access of upstream nodes will be higher than that of downstream nodes. The unfairness will become severe as the load of the network become heavy [13]. The problem had been fervidly explored [14-18] before and after the IEEE 802 committee recommended optical-bus distributed-control TDMA networks to form the IEEE 802.6 LAN/MAN protocol, which is named distributed-queue dual-bus (DQDB) networks, in 1990 [19]. Many approaches were proposed to improve the unfair problem [14-15], but it is yet to be resolved and has been laid for more than fifteen years.

The unfair distribution of access influences the distributedqueue delay of DQDB nodes [13]. In order to understand the characteristics of distributed-queue delays of DQDB nodes, several approaches were taken to analyse DQDB networks [16-18]. All these analyses are based on the model Bisdikian proposed [18]. Bisdikian presented an approximate singlenode analytical model. Due to the model, the steady-state generation function of the number of requests queued ahead of an arriving packet is expressed. Given this number, the distributed-queue delay of an arriving packet can be easily obtained. In a word, these approaches analyse the distributed- 
queue delay of every node according to the detailed operations of DQDB MAC protocol. Because the MAC protocol is so complex, the modelling and performance analysis of the network become very difficult $[11,17,18]$. To make an exact analysis on the distributed-queue delay by considering the detailed operations of DQDB MAC protocol is almost impossible [18]. Consequently, these analyses cannot make a contribution to resolve the unfair-access problem. Because users within a public subnetwork are independent of one another, it is impossible to deploy FTTH networks with the unfair-access problem.

Recently several structures of Ethernet passive optical networks (EPONs) [20-22] have been proposed for constituting FTTH networks in order to settle the urgent requirement on the bandwidth of access networks. Each structure uses tree or ring topology to provide the multi-access environment. In tree FTTH networks [20-21], an optical line terminal (OLT) within central offices (COs) is the root of a tree and sets of optical fibres are fanned out from a passive splitter to nodes. The splitter is considered as a branching point of a tree. An optical link between a root and a branching point is a trunk comprising upstream and downstream channels. The upstream and downstream channels are with TDMA and time-division multiplexing (TDM) respectively $[20,21]$. In ring FTTH networks [22], the ring is formed by a set of optical fibres which directly connects with a plurality of nodes. The ends of the ring terminate at an OLT within COs. TDMA and TDM flows on the ring optical fibres respectively represent the upstream and downstream channels.

Besides EPONs, the star topology was first adopted to deploy FTTH networks by Scandinavian countries. Now, the adoption of the star topology spread to the rest of Europe and the Middle East [23]. In the star topology, a CO and every customer's home are connected by a point-to-point (P2P) dual-fibre link. A CO is the centre of star topology. Because medium-sharing technology is not applied on the P2P link, every home can take more bandwidth for services but the bandwidth utility of the link will be low. The low bandwidth utility enlarges the number of optical fibres put into a $\mathrm{CO}$. The larger the number of optical fibres put into a $\mathrm{CO}$, the more the payment for the civil engineering distributing optical fibres to homes and the required real estate within central offices [23]. Otherwise, the delay for forwarding packages by exchanges will be lengthened as the number of optical fibres put into a $\mathrm{CO}$ becomes large. In practice, the constructing and maintaining costs of P2P FTTH networks are larger than that of FTTH EPONs [23]. This manifests that it is logical and necessary to deploy FTTH networks with medium-sharing technology.

Both tree and ring EPONs use centralized-control methods to distribute the upstream bandwidth to nodes [20, 22, 24-25]. For tree EPONs, the use of centralized-control methods can prevent collisions when several nodes send packets through splitters simultaneously [20, 24-25]. Within the OLT of EPONs, scheduling mechanisms, which carry out dynamic bandwidth allocation on upstream channels, allot transmissible bandwidth to a node based on both the upstream load of
EPONs and the bandwidth requested by customer premise equipments (CPEs) attached to the node [25]. Traffic management protocols, such as the multi-point control protocol (MPCP) recommended in IEEE 802.3ah, periodically interchange traffic information between an OLT and its attached nodes so as to accomplish dynamic bandwidth allocation [24]. In order to prevent the increase of bandwidth consumed by the MPCP, priority queues in splitters with logical link identifiers (LLIDs) are proposed to cooperate with the MPCP [24]. The downstream bandwidth is not dynamically allotted to nodes by OLTs. Messages carried by a TDM downstream channel are broadcasted to all nodes [25].

Because the upstream access is distributed to nodes by centralized-control methods, the upstream performance of every node is not correlative with nodal positions. Therefore, the unfair-access problem on optical distributed-control networks has disappeared on EPONs.

The scheduling mechanism within OLTs dynamically allocates nodal transmissible bandwidth according to the upstream load. The transmissible bandwidth of an originating node is periodically adjusted from the bandwidth requested by the node to two times the appointed bandwidth which is registered in the commercial contract of the node [25]. On TDM downstream channels, the available TDM bandwidth of a node is invariable and does not adapt to the downstream load. In a word, EPONs provide an asymmetric environment [23] whose upstream traffic is usually greater than their downstream traffic. However, for some broadband services applied by most customers, their downstream traffic is always much larger than their upstream traffic. As the bandwidth requisite for transporting downstream traffic of a node is much larger than the invariable TDM bandwidth of the node, longterm delays will take place. And the efficiency of EPONs for the node will be lowered. The larger the long-term delay, the lower the efficiency of EPONs.

On the other hand, scheduled holdups reduce the bandwidth utility of upstream and downstream channels. A scheduled holdup of a CPE is the duration from the CPE sends a request to take transmissible bandwidth to the $\mathrm{CPE}$ can write messages onto the upstream slots allotted to it. During scheduled holdups, nodes cannot write messages out even if upstream channels have empty slots. The larger the scheduled holdup, the lower the bandwidth utility on upstream and downstream channels.

So as to dynamically allocate bandwidth, it must consume a substantial portion of upstream and downstream bandwidths to periodically perform the MPCP. Therefore, the bandwidth that can carry payloads will be decreased. The situation influences the payload-to-capacity ratio. The payload-to-capacity ratio is the ratio of the bit rate of slots which carry payloads to channel capacity. The more the bandwidth consumed by the MPCP, the less the payload-to-capacity ratio.

On EPONs, downstream messages on rings are directly broadcasted to nodes. But that on trees are broadcasted to nodes through optical fibres fanned out from passive splitters. Only messages which are received by the destined node are the effective traffic of fanned-out optical fibres. Therefore, the 
bandwidth utility of fanned-out optical fibres will be very low. Otherwise, because splitters are passive, the number of fanned-out optical fibres must be restricted in order to prevent the performance of optical signals from degradation. Consequently, the bandwidth of upstream channels also cannot be sufficiently utilized.

Based on above discussions, EPONs use centralized-control methods to prevent the unfair-access problem. But the centralized-control procedure lowers the efficiency, the bandwidth utility, and the payload-to-capacity ratio of EPONs. These negative effects debase the merit of FTTH networks.

If optical-bus distributed-control TDMA networks could be adopted to deploy FTTH networks, all negative effects on FTTH EPONs can be excluded and the merit of FTTH networks will be more evident. In optical-bus distributedcontrol TDMA networks, all nodes are connected with the upstream and downstream channels directly. There are not low bandwidth-utility fanned-out optical fibres. Because of direct connections, every node can control itself to take the access of empty slots by its own MAC. So, there is not collision. In other words, the MAC of the network can apply distributedcontrol approaches to accomplish medium access so that packet collisions due to indirect connections will naturally disappear. Therefore, the mechanism requisite for performing dynamic bandwidth allocation and traffic management can be omitted. There are not scheduled holdups so that the bandwidth utility of upstream and downstream channels will be enhanced. Otherwise, the access of upstream and downstream channels can be separately distributed to nodes in accordance with customer's services. Thus both upstream and downstream channels can be used flexibly and efficiently in order that long-term delays due to invariable TDM bandwidth will not take place. Because it is not necessary to exploit the MPCP to perform traffic management, almost all slots can be occupied by payloads. The payload-to-capacity ratio of the network will approximate $100 \%$. The negative effects resulted from centralized control are vanished at all. However, the unfair-access problem on DQDB networks also accompanies optical-bus distributed-control TDMA networks.

Bandwidth is one of the most valuable resources for communications. In order to obtain an FTTH network capable of high bandwidth utility, it is a prerequisite to resolve the unfair-access problem on optical-bus distributed-control TDMA networks.

In 2007, the probability that how many nodes are simultaneously activated on an optical-bus distributed-control network is presented in [13]. Based on the probability and the simulations in [13], it is deduced that the average waiting time of distributed-control TDMA networks should be relative to nodal traffic. This deduction was successfully proved by analysing the waiting time of distributed-control TDMA nodes [26]. The analysis exhibits that the average waiting time of optical-bus distributed-control TDMA nodes is in inverse proportion to nodal traffic regardless of nodal positions. This analysis clearly and definitely manifests that optical-bus distributed-control TDMA networks are inherent in ideal fair behaviour when the MAC of their nodes performs traffic control. Due to the definite analytical result, a traffic-control method suitable to be applied on the optical-bus distributedcontrol networks is also presented [26]. The proposed trafficcontrol method can result in the ideal fair behaviour. The unfair-access problem on optical-bus distributed-control networks has been completely overcome. Therefore, the optical-bus distributed-control TDMA networks will be appropriately adopted to deploy FTTH networks.

In order for supporting full duplex service, two optical-bus distributed-control TDMA networks can be combined to constitute a bus FTTH network $[27,28]$. The MAC of the bus FTTH networks must perform traffic control so as to give rise to the ideal fair behaviour of networks. The structure of the bus FTTH network is so simple that its operations are easy and accessible for customers and operators. When all-fibre optical access networks are established, the merit of the bus FTTH networks will be more evident. In all-fibre optical access networks, every home is attached to a set of optical fibres regardless of the size of bandwidth the home requires.

In the next section, the structure of bus FTTH networks is presented. For FTTH deployment, the relationship between optical-bus distributed-control TDMA networks and public FTTH networks is described in Sec. III. The comparison among various medium-sharing FTTH networks is also included in this section. Conclusions are in the last section.

\section{BUS FTTH NETWORKS}

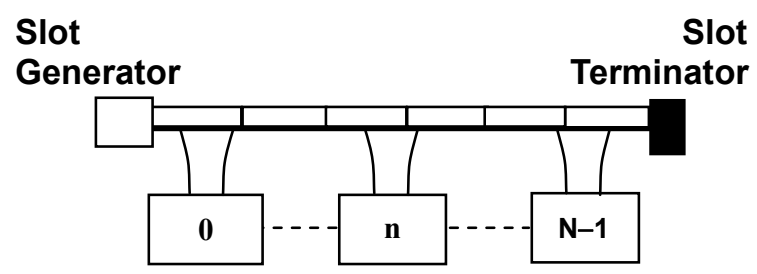

Figure. 1 Optical-bus distributed-control TDMA networks.

Fig. 1 shows the structure of optical-bus distributed-control TDMA networks. In the figure, the medium between the slot generator and the slot terminator is an optical fibre. The slot flow on the optical fibre is sent by the slot generator and sinks into the slot terminator. The number of nodes directly connects with the optical fibre. Nodes are numbered from 0 to $(\mathrm{N}-1)$ in sequent order. The ordinal number of every node also relates to the nodal position in the topology. The unfair access indicates that the node with small ordinal number has more access of TDMA slots on the optical fibre.

After exploiting the traffic-control MAC, the unfair problem on the network has been completely resolved. Two optical-bus distributed-control TDMA networks are combined to form a bus FTTH network whose structure is shown in Fig. 2.A pair of fibres is exploited in the structure. One is used as a transmitting bus (T Bus). The other is regarded as a receiving bus (R Bus). OLTs in the structure are partitioned into two parts. The first part located inside or near COs is called an OLTN (the OLT part near COs). The second part terminating fibres in the outside plant and far from $\mathrm{COs}$ is named an OLTF (the OLT part far from COs). 


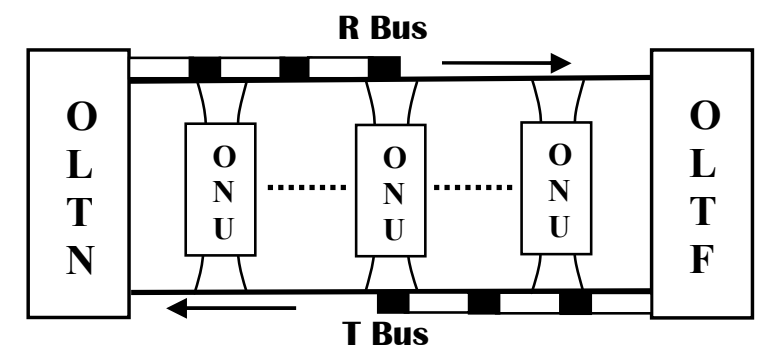

Figure. 2 The structure of bus FTTH networks.

Both the OLTN and OLTF comprise one slot generator and one slot terminator. The TDMA slot flow on the T bus is sent by the slot generator in the OLTF and sinks into the slot terminator in the OLTN. On the other hand, the TDMA slot flow on the R Bus is sent by the slot generator in the OLTN and sinks into the slot terminator in the OLTF. The T Bus is an upstream channel and the R Bus is a downstream channel. The OLTN transfers segments between the FTTH and backbone networks. The segments carried by slots on the $\mathrm{T}$ Bus are destined for customers supported by backbone networks. The segments in slots on the R Bus are received by nodes, which are named ONUs in the figure, attached to the FTTH network. The MACs of ONUs and OLTNs use trafficcontrol methods to accomplish medium access. A bus FTTH network established in an all-fibre optical access environment is shown in Fig, 3.

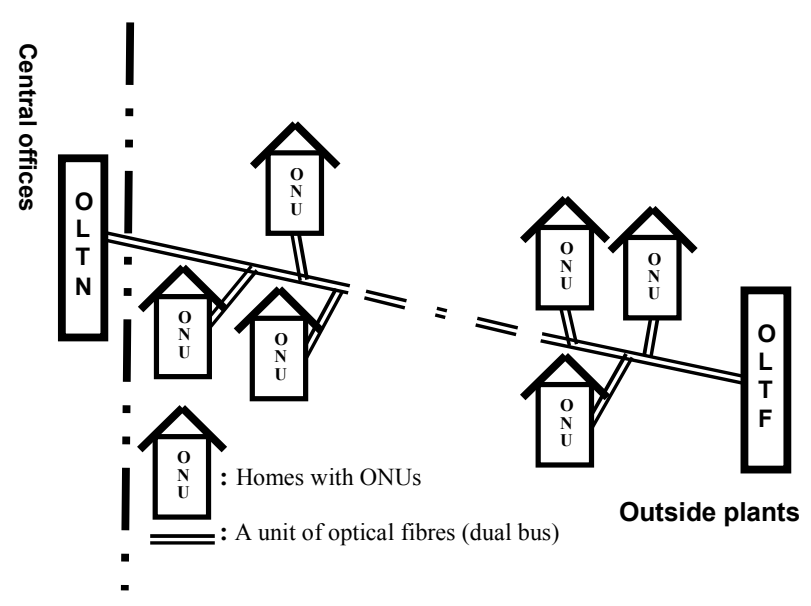

Figure. 3 Bus FTTH networks in an all-fibre optical access environment

\section{FOR FTTH DEPLOYMENT}

Public access networks can be considered as bridges between backbone networks and customers. Wider bridges can carry more traffic. Comparing optical fibres with twisted pairs, optical fibres can carry more traffic than twisted pairs. The required bandwidth of each customer is so small that the bandwidth of a set of optical fibres should be shared by lots of customers.

Due to the analysis of waiting times [26], optical-bus distributed-control TDMA networks whose MAC controls access with traffic-control approaches [26, 29] can be regarded as the subnetworks of public FTTH networks.

Because the access of the subnetwork is accomplished by traffic-control MACs, the medium-sharing network can naturally enhance bandwidth utility. Bus FTTH networks consisting of dual optical-bus distributed-control TDMA network can provide full duplex service. Due to the high capacity and good performance of optical networks, the bus FTTH network is appropriate to provide multimedia and broadband communications even if customers within the network spread over an extensive area. On the other hand, public FTTH networks are commercial networks. Customers of public networks pay for the bandwidth they require. Hence, a manager of public networks must stably and precisely provide a customer with access based on the commercial contract agreed by the customer. In relation to commercial contracts, the appointed bandwidth of a customer is known prior to the customer can take access. The appointed bandwidth of a customer can be transformed into the trafficcontrol MAC to control access. Therefore, the request sent to reserve slots can be omitted. And network managers can precisely provide appointed bandwidth for each customer regardless of the variation of nodal positions and carried load on bus FTTH networks.

As respects constructing and maintaining costs, it needs the least to deploy FTTH networks with distributed control and bus topology. The phenomenon will be more obvious as constructing all-fibre optical access networks in which every home is attached to a set of optical fibres regardless of the size of bandwidth the home requires.

When all-fibre optical access networks are instead of traditional twisted-pair access networks, the service area of a central office will be greatly enlarged and the number of central offices will become small. From the perspective of the topology of FTTH networks, the number of central offices whose FTTH networks are with tree or bus topology will be smaller than that with ring topology. The number of optical fibres required for constructing tree FTTH networks will be much larger than that required for constructing bus or ring FTTH networks. To construct central offices, it must pay a large cost for estates, civil engineering and exchanges. It also requires payment for civil engineering to distribute optical fibres to every home. The payment depends on the number of optical fibres. On the other hand, the maintaining cost will be increased when either the number of central offices or the number of optical fibres is enlarged. Therefore, the numbers of central offices and optical fibres can indirectly represent the constructing and maintaining costs for deploying FTTH networks. Reducing either the number of central offices or the number of optical fibres can lower the constructing and maintaining costs. When bus FTTH networks are deployed, the number of central offices and the number of optical fibres can be greatly reduced simultaneously. Consequently, the constructing and maintaining costs for deploying FTTH networks by bus topology will be suppressed more profusely than that by other topology. 
In respect of the unfair-access problem, the problem on bus FTTH networks had been completely resolved as the waiting time on optical-bus distributed-control TDMA networks was successfully analyzed. Because every node controls the access of empty slots by itself, it is not necessary to perform the MPCP which is exploited by EPONs. Therefore, the carried load of bus FTTH networks can approach the capacity of TDMA networks. And delays resulted from centralized control, such as scheduled holdups, are disappeared. For tree and ring FTTH EPONs, the unfair-access problem does not accompany these networks inherently, but the centralizedcontrol mechanism, which accomplishes the dynamic bandwidth allocation and the traffic management, has to be applied. The MPCP performing traffic management results in the centralized-control delay and bandwidth consumption in order to interchange traffic information between an OLT and its attached nodes. The dynamic bandwidth allocation periodically adjusts the transmissible bandwidth of nodes to increase the bandwidth utility of upstream channels. Therefore, in EPONs, the transmissible bandwidth of a node is usually greater than the transmitted traffic of the node and their difference is extra bandwidth, which is not requested by the node but additionally supplemented by the procedure of dynamic bandwidth allocation. However, the efficacy of unused bandwidth discussed by Chiou [30] manifests that the performance of a TDMA node varies dominantly with its transmitted traffic and is slightly affected by the extra bandwidth. To enlarge the extra bandwidth cannot enhance nodal performance without restraint [30]. Hence, most extra bandwidth has no efficacy on enhancing performance and is wasted. In other words, the larger the extra bandwidth, the more the wasted bandwidth on upstream channels of EPONs. Consequently, the bandwidth utility of upstream channels on tree or ring EPONs is much lower than that of bus FTTH networks. Otherwise, it also cannot provide a constant quality for service according to the periodic dynamic adjustment of nodal transmissible bandwidth.

Because the bandwidth of a bus FTTH network is directly shared by a plurality of customers, high bandwidth utility, which lowers both constructing and maintaining costs, will naturally take place. Due to the traffic-control MAC, the managers of public networks need not worry about the troublesome caused by the unfairness. Otherwise, the structure and operations of the bus FTTH network is so simple and its performance is so high that the network will be more accessible to operators and customers than EPONs.

\section{IV.CONCLUSIONS}

The paper introduces the bus FTTH network in order to deploying all-fibre optical access networks. The bus FTTH networks greatly lower the constructing and maintaining costs and increase the bandwidth utility of public access networks.

Recently EPONs have been proposed for constituting FTTH networks. The proposed EPONs use centralized-control methods to dynamically allocate the bandwidth of upstream TDMA channels. Due to the centralized control, EPONs does not accompany the unfair-access problem caused by topology but the bandwidth utility, the efficiency, and the payload-tocapacity ratio of FTTH EPONs are lowered. So as to get rid of these negative effects, it is necessary to deploy FTTH with bus distributed-control TDMA networks. But the unfair-access problem on distributed-control TDMA networks must be resolved first.

After the unfair-access problem on optical-bus distributedcontrol TDMA networks had been resolved, bus FTTH networks were proposed. In all-fibre optical access environment, the number of central offices and the number of optical fibres used to establish bus FTTH networks will be reduced more greatly than that used to establish FTTH EPONs. Due to the traffic-control MACs, the efficiency, the bandwidth utility, and the payload-to-capacity ratio on bus FTTH networks will be much better than that on centralized-control FTTH EPONs. Therefore, it can profusely suppress the constructing and maintaining costs to deploy FTTH with distributed control and bus topology.

Bus FTTH networks apply distributed-control mechanism to resolve the unfair-access problem. FTTH EPONs use centralized-control mechanism to prevent unfair access. Both bus FTTH networks and FTTH EPONs are inherent in the ideal fair behaviour of networks. The distributed-control mechanism on bus FTTH networks urges to evidently reveal the merit of FTTH networks, but the centralized-control mechanism causes the consumption and waste of bandwidth on FTTH EPONs.

\section{REFERENCES}

[1] "Next generation access networks - a unique opportunity for sustainable competition", European Telecommunications Network Operators' Association Press Corner, June 24, 2008.

[2] H. Shinohara, "Next generation networks: NTT's view and plan," in Proc. IEEE Int. Conf. Photonics in Switching, 19-22 Aug. 2007, pp.1-2.

[3] Batagelj, B., "FTTH networks deployment in Slovenia," in Proc. ICTON, 2009, pp. 1-4.

[4] van den Hoven, G., "FTTH deployment taking off in Europe,"in Proc. 5BROADNETS. 2008, pp. 221-221.

[5] Jaehyoung Park, Geun Young Kim, Hyung Jin Park, and Jin Hee Kim, "FTTH deployment status \& strategy in Korea: GW-PON based FTTH field trial and reach extension strategy of FTTH in Korea,"in Proc. GLOBECOM. 2008, pp. 1-3.

[6] K. Casier, B. Lannoo, J. Van Ooteghem, S. Verbrugge, D. Colle, M. Pickavet, and P. Demeester, "Game-theoretic optimization of a fiberto-the-home municipality network rollout," IEEE/OSA J., Optical Communications and Networking, vol. 1, no. 1, pp 30-42,. June 2009.

[7] B. Lannoo, K. Casier, J. Van Ooteghem, B. Wouters, S. Verbrugge, D. Colle, M. Pickavet, and P. Demeester, "Economic benefits of a community driven fiber to the home rollout,"in Proc., BROADNETS 2008., pp. 436-443.

[8] S. Verbrugge, K. Casier, B. Lannoo, J. Van Ooteghem, R. Meersman, D. Colle, and P. Demeester, "FTTH deployment and its impact on network maintenance and repair costs,"in Proc. ICTON, 2008, vol. 3, pp. 2-5.

[9] S. Kulkarni, B. Polonsky, and M. El-Sayed, "FTTH network economics: key parameters impacting technology decisions,"in Proc. 13th IEEE Int. Telecommunications Network Strategy and Planning Symp., Networks 2008. Sept. 28 2008-Oct. 2 2008, pp. 1-27.

[10] Peter Martini, "The DQDB protocol-What about fairness," in Proc. GLOBECOM, 1989, pp.298-302.

[11] M. Conti, E. Gregori, and L. Lenzini, "A methodological approach to an extensive analysis of DQDB performance and fairness," IEEE JSAC, vol.9, no.1, pp.76-87, 1991

[12] Peter Martini, "Fairness issues of the DQDB protocol," the 14th Conf. Local Computer Networks, 1989, pp.160-170. 
[13] R. N. Chiou, and J. K. Guo, "The effect of bursty lengths on DQDB networks," in Proc. CISIS'07, Vienna, Austria, 2007, pp.85-91.

[14] T. J. Kin, and D. H. Cho, "Bandwidth tuning for fairness of DQDB in client-server traffic environments," IEEE Communication Letters, vol.4, no.6, pp.208-210, June 2000.

[15] G. B. Brewster, and M. K. Vernon, "The fairness of DQDB networks with slot reuse," in Proc. INFOCOM, 1995, pp.1154-1163.

[16] C. Y. Roger Chen, Georges A. Makhoull, and Dikran S. Meliksetian, "A queueing analysis of the performance of the DQDB," IEEE Trans. Networking, vol.3, no. 6, pp.872-881, 1995.

[17] W. Jing, and M. Paterakis, "Appling the single-node DQDB analytical model to network-wide performance analysis," in Proc. GLOBECOM'9, 1992, pp.1618-1622.

[18] C. Bisdikian, "Waiting time analysis in a single buffer DQDB network," IEEE JSAC, vol.8, no.8, pp.1565-1573, 1990.

[19] IEEE Distributed Queue Dual Bus (DQDB) Subnetwork of a Metropolitan Area Network (MAN), IEEE Standard 802.6, 1990

[20] B. D. Edgar, "Method and apparatus for TDM/TDMA communications," U.S. Patent 6778 550, Aug.17, 2004.

[21] Singer Josef Dipl-Ing, "Method for addressing separate units in on optical TDM/TDMA system." European Patent 0665 669, Aug. 02 1995

[22] Kuo Jer-Chen and Pesavento Gerald A, "Passive optical network," U.S Patent 7272 321, Sep. 18, 2007

[23] Bostjan Batagelj, (2013) "Deployment of fiber-to-the-home in the Slovenian telecommunications market," Fiber and Integrated Optics, [Online]. 32(1), pp.1-11. Available: http://dx.doi.org/10.1080/01468030.2012. 760686

[24] Joseph G. DeCarolis, Chung Liang, Hanumanthrao NimishaKavi, "Enhanced upstream data traffic management for EPON," U.S. patent US 7639694 B2, Dec. 29, 2009.
[25] Yi-Ching Lian, Yun-Lung Chou, Zone-Chang Lai, Yeong-Chang Maa Chia-Chieh Tuanmu, Chu-Hsun Chang, "Methods for allocating transmission bandwidth of a network," US patent US 7577162 B2, Aug. 18, 2009.

[26] R. N. Chiou and C. N. Shyi, "Performance analysis on optical network with TDMA," in Proc. ICT, Marrakech, Morocco, 2009, pp.364-368.

[27] R.N. Chiou, "Bus-based optical network system," Japan Patent 5301 495, June 282013.

[28] Rong Nan Chiou, "TDMA FTTH networks with bus topology," Journal of Telecommunications. vol.11 issue 2, pp. 71-75, December 2011

[29] R.N. Chiou, "Medium access control methods for optical-fiber TDMA networks," U.S.A. Patent 8218970 B2, July 102012.

[30] Rong Nan Chiou, (2011 Dec.) "The efficacy of unused bandwidth in the performance of TDMA networks," Journal of Telecommunications. [Online], 11(2), pp.65-70.

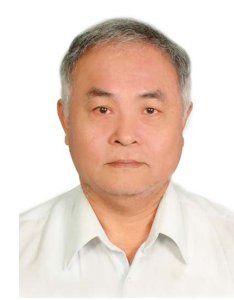

Rong Nan Chiou (M'11) was born in Tainan City, Taiwan on September 20, 1951.He received the B.S. M.S., and Ph.D. degrees in electrical engineering from National Cheng Kjug University, Tainan, Taiwan, in 1985, 1988, and 1993, respectively.

From 1975 to 1999 , he was with the South Taiwan Telecommunication Administration, working with switching systems and data communications. He was an Associate Professor of electronic engineering at Kun Shan University, Tainan, Taiwan from 2000 to 2007. He is retiring since 2007. His current research interests include networking and data communications. 